\title{
Potential Pitfalls for False Suspicion of Papillary Thyroid Carcinoma: A Cytohistologic Review of 22 Cases
}

\author{
Xin Jing, M.D. ${ }^{*}$ and Claire W. Michael, M.D.
}

To evaluate the diagnostic pitfalls attributing to false suspicious interpretation of papillary thyroid carcinoma (PTC), we performed a retrospective cytohistologic review of thyroid nodules diagnosed as suspicious for papillary carcinoma with histologyproven false suspicion of PTC. A total of 22 thyroid fine needle aspirates along with the corresponding surgical specimens were reviewed. The presence and/or absence of PTC-associated morphologic features and their mimics were evaluated. One aspirate showed papillary-like fragments with honeycomb architecture. One aspirate contained rare syncytial-type fragments which were accompanied by honeycomb sheets of follicular cells and moderate amount of colloid. Intranuclear grooves presented occasionally in virtually all 22 aspirates. The grooves were noted mainly within round nuclei and appeared thin and/or incomplete. Rare intranuclear pseudoinclusions were detected in one otherwise benign-appearing aspirates. Eight aspirates contained various numbers of histiocytes, as well as atypical polygonal, epithelioid, elongate, or spindle cells in which intranuclear grooves were occasionally seen. Conspicuous fragments of fibrocollagenous tissue with entrapped follicular cells were detected in one aspirate. The corresponding histological specimen revealed counterpart of the cytological findings described. Other features associated with PTC were inconspicuous. The findings demonstrated that the following factors may contribute to the false suspicion of PTC: (1) misinterpretation of papillary-like tissue fragments with honeycomb arrangement and fragments of fibrocollagenous tissue with entrapped follicular cells; (2) overinterpretation of suboptimal intranuclear grooves and rare intranuclear pseudoinclusion in the absence of the other features of PTC; (3) misinterpretation of the polygonal, epithelioid,

Department of Pathology, University of Michigan, Ann Arbor, Michigan

*Correspondence to: Xin Jing, M.D., Department of Pathology, University of Michigan, 1500 E. Medical Center Drive, 2G332 UH, Ann Arbor, MI 48109-0054. E-mail: xinjing@med.umich.edu

Received 20 January 2011; Accepted 27 March 2011

DOI 10.1002/dc.21726

Published online 11 May 2011 in Wiley Online Library (wileyonlinelibrary. com). elongate, or spindle cells that actually represented atypical cyst lining cells. Diagn. Cytopathol. 2012;40:E74-E79. @ 2011 Wiley Periodicals, Inc.

Key Words: thyroid; suspicious for papillary thyroid carcinoma; cytohistologic discrepancy

It has been reported that an accuracy level of greater than $90 \%$ may be achieved utilizing fine needle aspiration (FNA) to diagnose papillary thyroid carcinoma (PTC). ${ }^{1}$ Although cytomorphological features associated with PTC have been well defined, none of the features alone is considered diagnostic for PTC. A category of "suspicious for PTC" has been used for the aspirates with features that raise concerns for PTC. The aspirates that are categorized as "suspicious for PTC" represent $2.4-8 \%$ of the total thyroid FNA aspirates and further, follow-up histologic examination reveals PTC in 60$80 \%$ of the suspicious aspirates. ${ }^{1,2}$ The recently published "The Bethesda System for Reporting Thyroid Cytopathology" (BSRTC) demonstrates the criteria of "suspicious for malignancy" and "suspicious for PTC" represents the majority of the lesions falling into suspicious category. BSRTC recommends judicious use of this diagnostic category so that an ideal positive predictive value can be achieved. ${ }^{3}$ Generally, the presence of overlapping cytological features between PTC and nonPTC, as well as the over interpretation of certain PTCassociated features are briefly mentioned as contributing factors to cytohistologic discrepancy. There are limited studies that specifically address details of cytologic pitfalls associated with cytohistologic discrepancy. This study was conducted through a retrospective cytohistologic review to assess the cytomorphologic features that 
contributed to false suspicion of PTC and describe the potential pitfalls for misinterpretation.

\section{Materials and Methods}

Between January 2000 and June 2010, a total of 4,563 thyroid FNAs were performed in our institution. A SNOMED search of the electronic pathology database retrieved a total of 28 thyroid aspirates in which a cytologic interpretation of "suspicious for PTC" was previously rendered and the evidence of PTC was not found upon examining the histologic specimens of subsequent total thyroidectomies. Both cytologic and histologic materials were available for a retrospective review in 22 of the 28 aspirates. All of the 22 aspirates consisted of Diff Quik- and Papanicolaou-stained conventional smears and in some aspirates also ThinPrep or cell block slides were available. In conjunction with review of several reference aspirates with histology-proven PTC, the two cytopathologists (X.J. and C.W.M.) simultaneously reviewed all aspirates along with the corresponding surgical specimens using a double-headed light microscope. During the review, we evaluated and recorded the presence and/or absence of morphologic features associated with PTC. ${ }^{3}$ These features included papillae and/or papillary-like (without central cores) fragments, syncytial-type sheets, swirling sheets, enlarged nuclei, irregularly shaped nuclei, molded nuclei, longitudinal intranuclear grooves, intranuclear pseudoinclusions, pale nuclei with powdery chromatin, marginally placed micronucleoli, Psammoma bodies, and multinucleated giant cells. In addition, morphologic findings that may resemble or mimic the PTC-associated features aforementioned were also assessed and recorded.

\section{Results}

All 22 aspirates previously interpreted as "suspicious for PTC" were adequate for cytologic assessment. The corresponding histology of the 22 aspirates revealed 16 nodules with nodular hyperplasia and six nodules with follicular adenoma. Ten aspirates were moderately cellular and 12 aspirates were hypocellular. Ten aspirates contained moderate amount of colloid. Scant or no colloid presented in the remaining 12 aspirates. Various proportions of monolayer honeycomb sheets, intact follicles, and microfollicles were appreciated among all aspirates. The occurrence of PTCassociated features and their mimics is summarized in Table I. Accordingly, one aspirate showed papillary-like (without central cores) fragments. The round or oval shaped nuclei within the fragments were evenly distributed in a honeycomb rather than syncytial pattern. Smooth nuclear membrane, fine chromatin, and occasional nucleoli were appreciated (Figs. 1A and B). One aspirate contained rare syncytial-type fragments (Fig. 1C) which were accompanied by honeycomb sheets of follicular cells and moderate amount of colloid. Intranuclear grooves presented occasion-
Table I. Cytomorphological Features in 22 Aspirates

\begin{tabular}{lc}
\hline Features & Number of cases/total \\
\hline Papillae with central cores & $0 / 22$ \\
Papillary-like fragments without central cores & $1 / 22$ \\
Syncytial-type sheets & $1 / 22$ \\
Swirling sheets & $0 / 22$ \\
Nuclear molding & $0 / 22$ \\
Nuclear enlargement & $0 / 22$ \\
Irregular nuclear membrane & $0 / 22$ \\
Intranuclear grooves & $22 / 22$ \\
Intranuclear pseudoinclusions & $1 / 22$ \\
Cystic degeneration & $8 / 22$ \\
Fibrocollagenous fragments & $1 / 22$ \\
Psammoma bodies & $0 / 22$ \\
Multinucleated giant cells & $0 / 22$ \\
\hline
\end{tabular}

ally in virtually all 22 aspirates. The grooves were noted mainly within round nuclei and appeared thin and/or incomplete (Fig. 1D). Rare intranuclear pseudoinclusions (1E) were detected in one otherwise benign-appearing aspirates. Eight aspirates contained various amount of histiocytes, as well as atypical polygonal, epithelioid, elongate, or spindle cells in which nuclear enlargement, intranuclear grooves, pale chromatin, and small distinct nucleoli were occasionally seen (Fig. 1F). Conspicuous fragments of fibrocollagenous tissue with entrapped follicular cells (Fig. $1 \mathrm{G})$ were detected in one aspirate. Review of the corresponding histological specimens revealed focal changes that correlated with the cytological findings described but were insufficient for the diagnosis of PTC. Examples are shown in Figs. 1H-K. The presence of mild nuclear atypia including nuclear molding, nuclear enlargement, irregular nuclear membrane, and nuclear pallor was minimal among all aspirates. Swirling sheets, Psammoma bodies, and multinucleated giant cells were inconspicuous.

\section{Discussion}

This retrospective review of 22 thyroid aspirates with a histology-proven false suspicious diagnosis of PTC demonstrated that the following factors were the main contributing features to the false suspicion of PTC: (1) misinterpretation of papillary-like tissue fragments with honeycomb arrangement or fragments of fibrocollagenous tissue with entrapped follicular cells; (2) overinterpretation of suboptimal intranuclear grooves in the nuclei with minimal diagnostic features of PTC; (3) overinterpretation of rare intranuclear pseudoinclusion in the absence of the other features of PTC; (4) misinterpretation of the elongate or spindle cells that actually represented atypical cyst lining cells.

Table II outlines differentiating features between true and false suspicion of PTC. Basically, papillary-like (without central cores) architecture may be seen not only in PTC but also in benign conditions such as nodular hyperplasia and Hashimoto's thyroiditis. In benign conditions, papillary-like fragments revealed honeycomb pat- 
JING AND MICHAEL
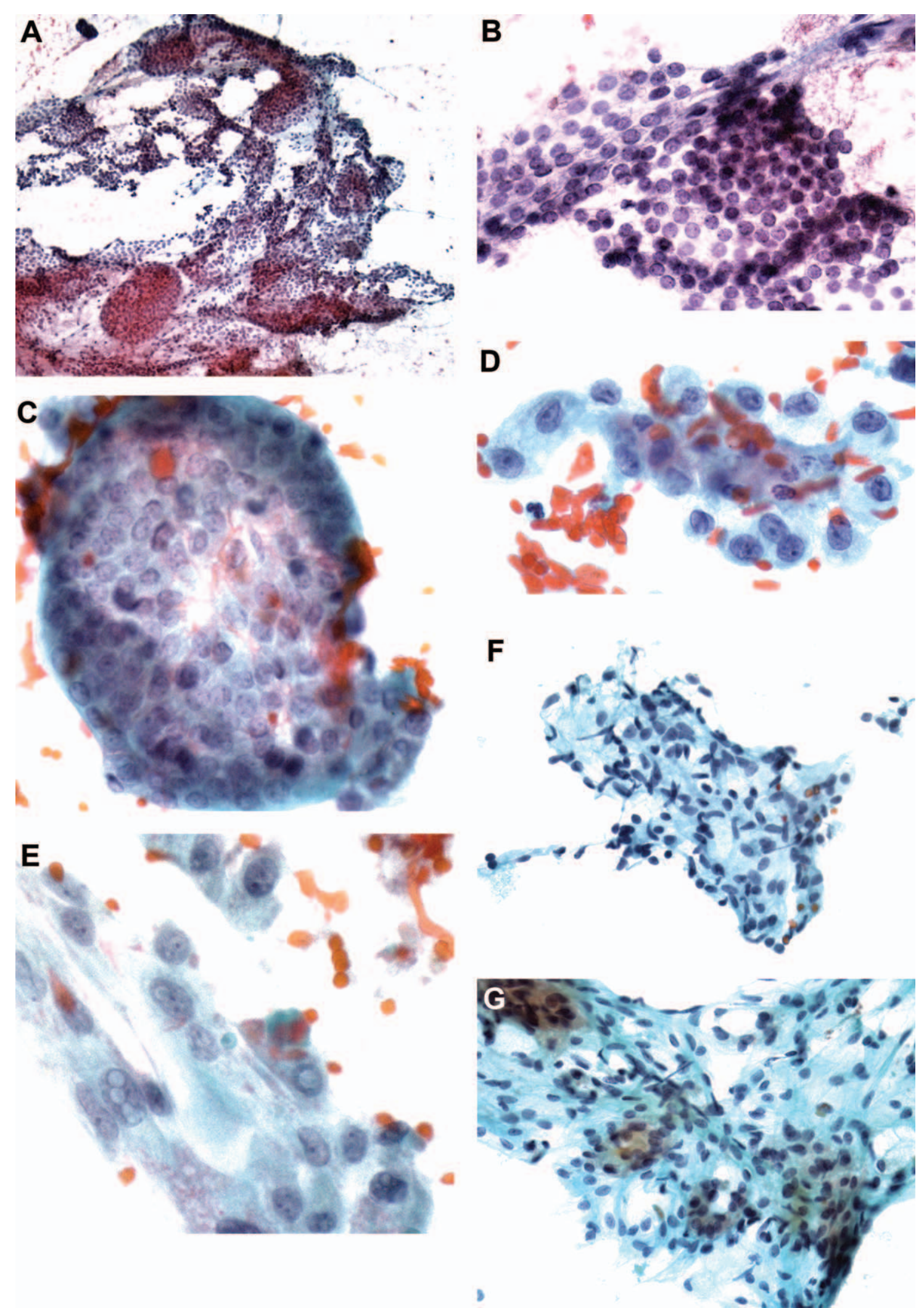

Fig. 1. PTC-associated cytologic features and their mimics. (A and B) papillary-like fragments with honeycomb arrangement (Papanicolaou, 100 $\times$ and $400 \times$ ); (C) syncytial-type fragments (Papanicolaou, 600×); (D) intranuclear grooves (Papanicolaou, 600×); (E) intranuclear inclusions (Papanicolaou, 600×); (F) cyst lining cells (Papanicolaou, 400×); (G) fragments of fibrocollagenous tissue (Papanicolaou, 400×); (H, I, J, and K) corresponding histology of (D), (E), (F), and (G) (H\&E, 200×). 

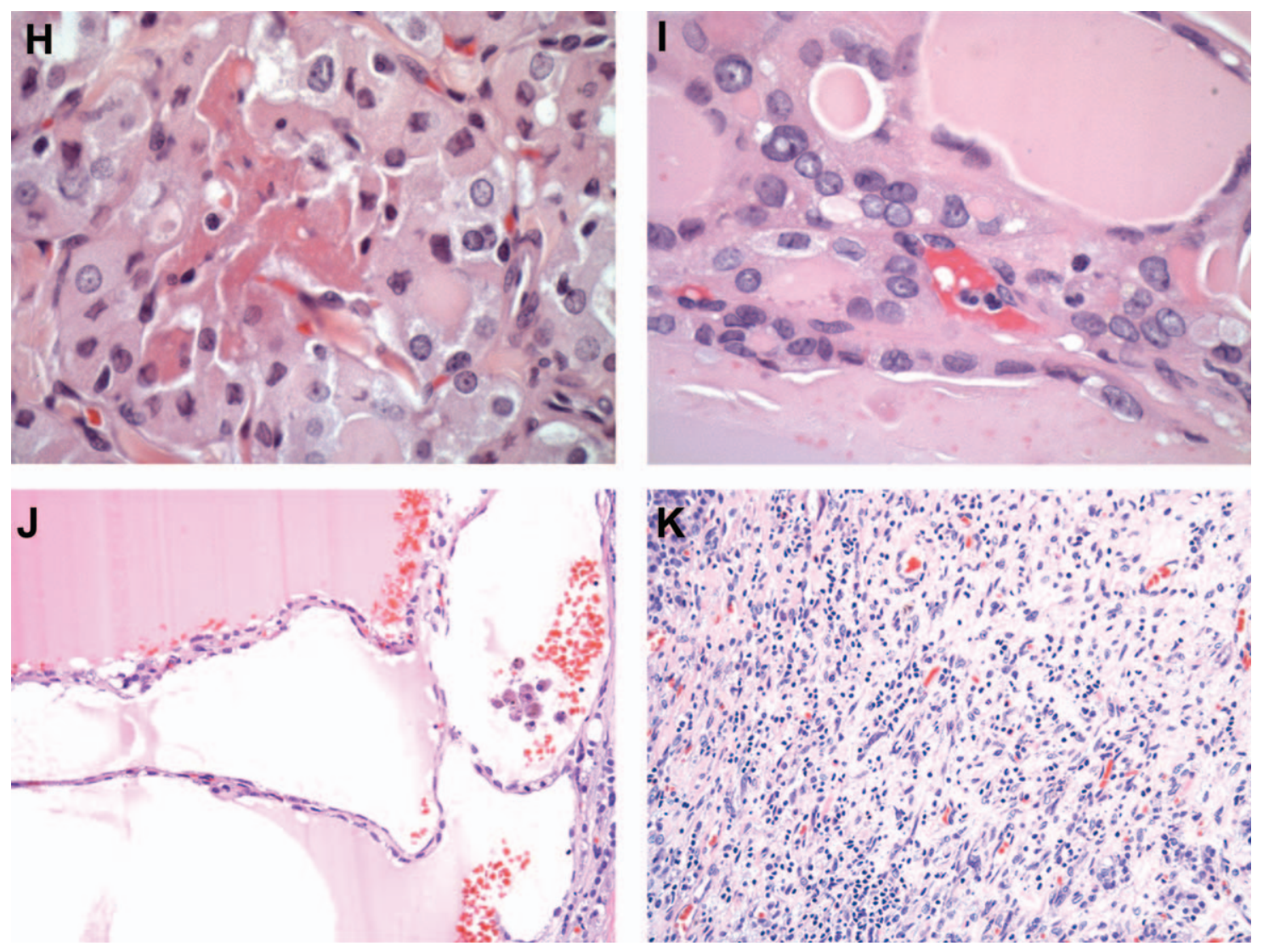

Fig. 1. (Continued).

Table II. Cytomorphologic Differentiation Between True PTC and False Suspicion of PTC

\begin{tabular}{lll}
\hline Features & \multicolumn{1}{c}{ True PTC } & False suspicion of PTC \\
\hline Papillary-like fragments & $\begin{array}{c}\text { Syncytial arrangement with nuclear crowding/ } \\
\text { overlapping/molding } \\
\text { Moderate to abundant, diffuse } \\
\text { Oval, elongate or irregular shaped, variation in size, } \\
\text { markedly enlarged, irregular contour, } \\
\text { pale, chromatin } \\
\text { Thick/longitudinal, accompanied by other } \\
\text { architectural/nuclear atypia }\end{array}$ & $\begin{array}{l}\text { Honeycomb arrangement with evenly spaced nuclei } \\
\text { Round or oval-shaped, uniform in size slightly } \\
\text { enlarged, smooth contour, finely granular chromatin }\end{array}$ \\
Intranuclear grooves & $\begin{array}{c}\text { Present along with other architectural/Nuclear atypia } \\
\text { Intranuclear pseudoinclusion }\end{array}$ & Thin/incomplete, an isolated finding \\
\hline
\end{tabular}

tern with well-defined cytoplasmic borders and evenly distributed nuclei. Features of nuclear atypia are inconspicuous or minimally visible. On the other hand, papillary fragments encountered in PTC exhibit syncytial arrangement with unevenly distributed nuclei, nuclear crowding, and/or nuclear overlapping. In addition, nuclear features of PTC are easily appreciated. ${ }^{4}$ Misinterpretation of honeycomb sheets as syncytial-type sheets could be avoided by performing mid-high power microscopic examination. Similarly, in the absence of definitive nuclear features of PTC, fragments of fibrocollagenous tissue with entrapped follicular cells should be interpreted with cau- tion so that the regenerative scarring or fibrotic components are not mistaken for fibrovascular cores of papillae of PTC.

Although the presence of intranuclear grooves is considered an important criterion in the cytological diagnosis of PTC, it could also be seen in non-PTC conditions including benign entities. The previously reported data indicated that PTC in comparison with other conditions showed higher number of cells possessing intranuclear grooves. Thus, the presence of intranuclear grooves in a widespread pattern can be considered a reliable criterion while the presence of occasional intranuclear grooves 
should be interpreted as a non-specific finding..$^{5-7}$ Virtually all 22 aspirates reviewed in this study showed focal intranuclear grooves and some grooves had thin and/or incomplete appearances. The latter was also noted by other investigators. We agree with the opinion that thin and/or incomplete lines are probably artifacts of chromatin alignment rather than true grooves. However, true groove and its mimics should be interpreted in conjunction with other features associated with PTC. ${ }^{5}$

The presence of intranuclear pseudoinclusions is considered another hallmark of PTC and can be seen in benign conditions. In this regard, Kini reported three aspirates obtained from Hashimoto's thyroiditis that contained intranuclear pseudoinclusions. ${ }^{4}$ Similarly, in one of our cases, we appreciated the presence of intranuclear pseudoinclusions as the sole atypical features suggestive of PTC and the corresponding histology revealed nodular hyperplasia.

Faquin et al. undertook a comprehensive study to examine cytohistologic feature of thyroid cyst with "atypical" cells. Accordingly, the "atypical" cells demonstrated a cytomorphologic spectrum from spindle, elongated cells to polygonal, epithelioid cells. More than 50\% of the aspirates showed the following features including nuclear enlargement, nuclear grooves, fine chromatin, and distinct nucleoli. Nuclear crowding and intranuclear pseudoinclusion were rarely detected. With the help of immunostaining, the "atypical" cells were proven to be cyst lining cells of follicular origin. ${ }^{8}$ Among the 22 aspirates included in this study, the "atypical" cyst lining cells were appreciated in eight aspirates which contained various number of histiocytes. For the purpose of this discussion, immunostaining for thyroglobulin, TTF-1, and CD 68 were performed on the corresponding surgical specimen and not surprisingly, histiocytes were highlighted by positive CD 68 staining while the cyst lining cells were positive for TTF-1 (Fig. 2) and thyroglobulin (not shown), confirming thyroid follicular origin. Although the presence of nuclear enlargement, pale chromatin, and occasional intranuclear grooves in the "atypical" cells is a worrisome finding for PTC, the suspicious diagnosis of PTC should be made with great caution due to the lack of other features associated with PTC such as nuclear crowding/overlapping, intranuclear pseudoinclusions, etc. Noting lower cellularity of the "atypical" cells in a benign background, it is very unlikely that the spindle-shaped "atypical" cells are misinterpreted as other malignant tumors such as medullary thyroid carcinoma, anaplastic carcinoma, and metastatic carcinoma. It is well known that spindle cells of medullary carcinoma usually have eccentric nuclei, coarser, or salt-and-pepper chromatins and indistinct nucleoli while anaplastic carcinoma and metastatic carcinoma exhibiting obvious cytomorphologic features of malignancy and/or relevant clinical history, which were absent in our cases.
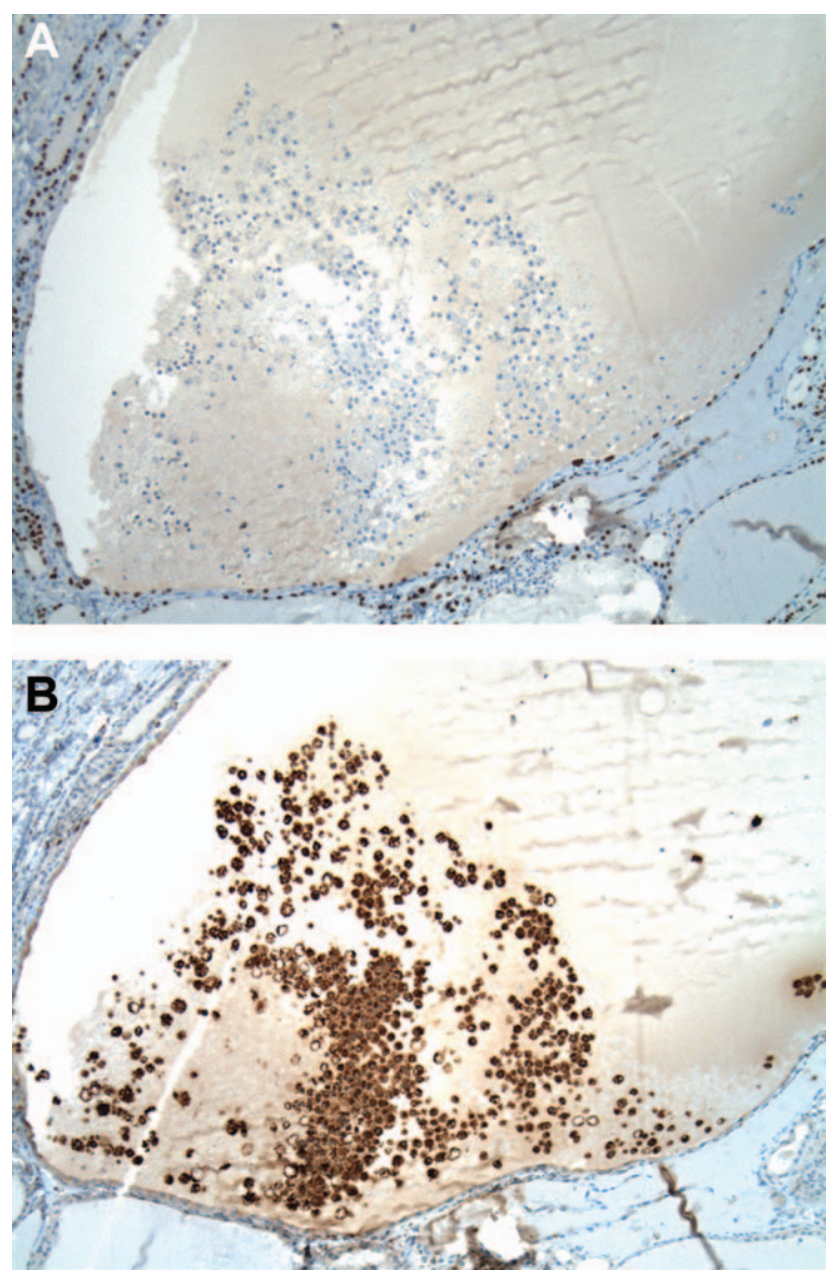

Fig. 2. Immunostaining for TTF-1 (A) and CD68 (B) (immunostain, $100 \times)$.

It is recognized that threshold and stringency of applying the criteria differ among reviewers in cytologic diagnosis of problematic thyroid nodules. The BSRTC recommends the use of "atypia of undetermined significance/ follicular lesion of undetermined significance (AUS/ FLUS)" for aspirates in which focal features of PTC, atypical cyst lining cells are seen in an otherwise predominantly benign setting. ${ }^{9}$ However, challenge does exist in routing cytologic practice with regard to separation of "suspicious for PTC" from "AUS/FLUS." Weber et al. ${ }^{10}$ reported histology-proven PTC in 25 of 57 aspirates which were interpreted as "atypical epithelial cells, cannot exclude papillary thyroid carcinoma." The blind, retrospective review of 123 aspirates which were originally interpreted as AUS/FLUS and followed by surgeries in our institution re-classified two aspirates as PTC which were confirmed by the corresponding surgical specimens while three cases of histology-proven PTC were still not diagnosed despite the secondary review. ${ }^{11}$ In conclusion, 
it is essential to recognize that the presence of focal atypical features that are reported in PTC could be recognized in non-PTC FNAs and are not sufficient to raise the suspicion for PTC unless other corroborating features are also noted. We recommend that such cases be reported as AUS/FLUS which will allow the patients the benefit of a repeat FNA to further evaluate the lesions.

\section{References}

1. Renshaw AA. Accuracy of thyroid fine-needle aspiration using receiver operator characteristic curves. Am J Clin Pathol 2001;116: 477-482.

2. Yassa L, Cibas ES, Benson CB, et al. Long-term assessment of a multidisciplinary approach to thyroid nodule diagnostic evaluation. Cancer 2007;111:508-516.

3. Wang HH, Filie A, Clark DP, Powers CN. Suspicious for malignancy. In: Ali SZ CE, editor. The Bethesda system for reporting thyroid cytopathology. Definitions, criteria and explanatory notes. New York: Spinger Science + Business Media, LLC; 2010. p 75-89.

4. Kini SR. Thyroid cytopathology: An atlas and text. Philadelphia: Lippincott Williams \& Wilkins; 2008. p 196-210.
5. Gould E, Watzak L, Chamizo W, Albores-Saavedra J. Nuclear grooves in cytologic preparations. A study of the utility of this feature in the diagnosis of papillary carcinoma. Acta Cytol 1989;33: 16-20.

6. Rupp M, Ehya H. Nuclear grooves in the aspiration cytology of papillary carcinoma of the thyroid. Acta Cytol 1989;33:21-26.

7. Francis IM, Das DK, Sheikh ZA, Sharma PN, Gupta SK. Role of nuclear grooves in the diagnosis of papillary thyroid carcinoma. A quantitative assessment on fine needle aspiration smears. Acta Cytol 1995;39:409-415.

8. Faquin WC, Cibas ES, Renshaw AA. "Atypical" cells in fine-needle aspiration biopsy specimens of benign thyroid cysts. Cancer 2005; 105:71-79.

9. Krane JF, Nayar R, Renshaw AA. Atypia of undetermined significance/follicular lesion of undetermined significance. In: Ali SZ, Cibas ES, editors. The Bethesda system for reporting thyroid cytopathology. New York: Springer; 2010. p 37-49.

10. Weber D, Brainard J, Chen L. Atypical epithelial cells, cannot exclude papillary carcinoma, in fine needle aspiration of the thyroid. Acta Cytol 2008;52:320-324.

11. Jing X, Roh MH, Knoepp SM, Zhao L, Michael CW. Minimizing the diagnosis of "follicular lesion of undetermined significance" and identifying predictive features for neoplasia. Diagn Cytopathol 2010. Oct 14. [Epub ahead of print]. 